\title{
Effect of the surface processing on the secondary electron yield of Al alloy samples
}

\author{
D. R. Grosso, ${ }^{1}$ M. Commisso, ${ }^{1}$ R. Cimino, ${ }^{1}$ R. Larciprete,,${ }^{1,2}$, R. Flammini,,${ }^{1,3}$ and R. Wanzenberg ${ }^{4}$ \\ ${ }^{1}$ LNF-INFN, via E. Fermi 40, 00044 Frascati (Roma) Italy \\ ${ }^{2}$ CNR-Institute for Complex Systems, via Fosso del Cavaliere 100, I-00133 Roma, Italy \\ ${ }^{3}$ CNR- Istituto di Metodologie Inorganiche e Plasmi, Via Salaria Km. 29.300, I-00019, Monterotondo Scalo, Italy \\ ${ }^{4}$ DESY, Notkestrasse 85, 22603 Hamburg, Germany
}

(Received 7 May 2012; published 28 May 2013)

\begin{abstract}
In this study we have investigated the relation between the secondary electron yield (SEY) and the surface chemical state for technical Al alloy samples cut from the inner walls of the Petra III storage ring. SEY curves measured after prolonged electron beam irradiation at $500 \mathrm{eV}$ showed maximum values $\left(\delta_{\max }\right)$ between 1.8 and 1.5. By combining x-ray photoelectron spectroscopy with SEY measurements, we have been able to relate the surface chemical composition to the $\delta_{\max }$ values for the "as-received" surface $\left(\delta_{\max }=2.7\right)$, for the electron beam conditioned sample $\left(\delta_{\max }=1.8-1.5\right)$, and after substantially removing the surface contaminating layer by means of $\mathrm{Ar}^{+}$ion sputtering $\left(\delta_{\max }=1.3\right)$. Our detailed chemical analysis shows that the SEY strongly increases in the presence of the thin surface oxide film which unavoidably forms on the clean $\mathrm{Al}$ alloy sample under electron beam irradiation even in ultrahigh vacuum conditions, and suggests that the high reactivity of pure $\mathrm{Al}$ and $\mathrm{Al}$ alloys to oxygen could be the cause of the difference among the SEY values measured in different ultrahigh vacuum environments.
\end{abstract}

DOI: 10.1103/PhysRevSTAB.16.051003

PACS numbers: 29.27.- a, 81.65. - b, 82.80.Pv

\section{INTRODUCTION}

For several years now, a growing number of observations of the electron cloud (EC) effects have been recorded in several high-energy particle accelerators [1-4]. The term EC refers to the accumulation of electrons inside an accelerator beam pipe capable of affecting the accelerator operation by causing emittance growth, vacuum pressure increase, and possibly beam loss and degradation of the beam diagnostics. During operation, the internal walls of the particle accelerators are exposed to synchrotron radiation and/or electron bombardment that can cause desorption of gases and production of primary electrons. These may be accelerated by the Coulomb potential of the circulating beam producing secondary electrons and leading to EC buildup [1-4].

At the synchrotron radiation facility PETRA III at DESY, the measured tune spectra show beam characteristics typically observed in connection with EC effects [5]. The estimation of such effects with the available EC simulation codes requires a clear determination of the secondary electron yield (SEY) of the chamber wall [5]. Furthermore it is crucial to investigate whether EC effects induce a conditioning of the accelerator environment, which changes the chemical state of the chamber surface and leads to a permanent lower SEY [6-13].

\footnotetext{
*Corresponding author. rosanna.larciprete@isc.cnr.it

Published by the American Physical Society under the terms of the Creative Commons Attribution 3.0 License. Further distribution of this work must maintain attribution to the author(s) and the published article's title, journal citation, and DOI.
}

PETRA III operates a positron beam in a primary chamber made of AlMgSi0,5 (6060), a technologically important $\mathrm{Al}$ alloy used to build vacuum chambers as well as critical inner components of particle accelerators. The investigations carried out so far on technical Al samples have emphasized the impossibility to obtain surfaces with a low SEY even after extensive conditioning [14,15], indicating the difficulty to modify in the desired direction the state of the $\mathrm{Al}$ surface via the impact of charged particles. On the other hand the large spread of the $\delta_{\max }$ values reported in the literature for different $\mathrm{Al}$ and $\mathrm{Al}$ alloys [6,8,14-16] ( $\delta_{\max }$ being the maximum of the measured SEY curves) denotes the importance of the chemical state of the metal surface, but also to the properties of the specific alloy, in determining the secondary electron yield. This poses a crucial issue as the simulation codes developed to correctly analyze, predict, and possibly mitigate the EC-related unwanted effects for PETRA III necessitate, among other input parameters, a reliable $\delta_{\max }$ value.

The most consistent way to face this need is via an ad hoc investigation aimed at determining the SEY of the accelerator walls. This is done by measuring the SEY and the chemical surface composition of an $\mathrm{Al}$ alloy sample as similar as possible to the actual ring wall, in correspondence of those surface modifications expected to occur during machine operation ( $e^{-}$and ion bombardment). Therefore, in this study, representative samples cut from a prototype dipole chamber of Petra III at DESY were studied by combining the measurement of the SEY curves with the surface chemical analysis obtained by $\mathrm{x}$-ray photoelectron spectroscopy (XPS) with two principal objectives: (i) to provide a reliable range of values for $\delta_{\max }$ as input value for the ECLOUD simulation code [17], and (ii) to 
relate the evolution of $\delta_{\max }$ to controlled treatments of the Petra III sample capable to alter its surface chemical state. To this aim the data taken on the "as-received" samples were compared with those measured after electron beam irradiation, performed to simulate the effect of the EC, as well as with the curves obtained for the $\mathrm{Ar}^{+}$ion sputtered and for the oxidized $\mathrm{Al}$ alloy surface. The possibility to relate the measured SEY to the chemical composition of the sample surface has revealed the critical impact that adsorbates and contaminants have on the secondary emission properties of such an Al-based alloy.

\section{PETRA III}

PETRA III [18] is a third-generation synchrotron radiation facility at DESY. The PETRA ring was built in 1976 as an electron and positron collider and used as a preaccelerator for the HERA lepton hadron collider ring from 1988 until 2007. During the conversion to a synchrotron radiation facility from 2007 to 2009 one octant of the PETRA ring has been completely redesigned to provide space for 14 undulators. Furthermore the whole vacuum system $[19,20]$ was replaced while the dipole and quadrupole magnets in seven octants were reused. The end section of the dipole vacuum chamber is shown in Fig. 1. Synchrotron radiation light impinges on the internal part of the vacuum chamber, as indicated by the white arrow. This chamber, made of an extruded aluminum alloy profile, is $5.8 \mathrm{~m}$ long and is used in the dipoles of the seven octants. A nonevaporable getter ( $\mathrm{Zr}, \mathrm{Ir}, \mathrm{V}$ alloy) strip is installed in a side channel of the aluminum alloy profile to provide distributed pumping along the chamber. Additionally two ion pumps are installed at each end of the chamber [20].

The PETRA III vessel was only prebaked by flowing $150{ }^{\circ} \mathrm{C}$ steam in the water cooling channels of the dipole chamber prior to installation [20]. After that the PETRA III chamber is not baked with external heaters but synchrotron radiation is used to perform the conditioning of the vacuum system [20]. In March 2012 the typical pressure measured at the ion pumps at the end of the dipole chambers was

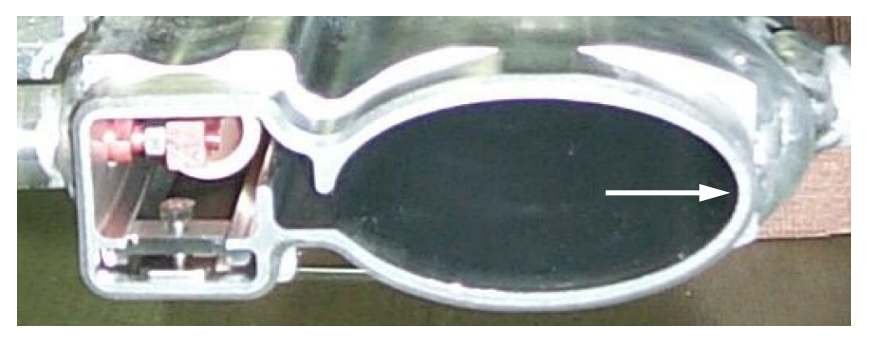

FIG. 1. PETRA III dipole vacuum chamber. The end of the chamber is shown with the ante chamber at the left side. A NEG strip is mounted in the ante chamber. The full width of the elliptical chamber is $80 \mathrm{~mm}$ and the full height is $40 \mathrm{~mm}$. The arrow indicates where the primary synchrotron radiation is hitting the chamber wall.
$6 \times 10^{-10}$ mbar without beam and about $3 \times 10^{-9} \mathrm{mbar}$ with beam $(100 \mathrm{~mA})$.

Commissioning with the beam started in April 2009 and user runs were started in 2010 [21]. PETRA III is presently running in a top-up operation mode with positrons since it is sharing the same preaccelerator chain with the synchrotron source DORIS, which is running with positrons to avoid problems with ionized dust particles. The main design parameters are listed in Ref. [18].

The design current of $100 \mathrm{~mA}$ has been achieved but using different filling schemes than originally foreseen, since a vertical emittance blowup has been observed for a filling scheme with 960 equidistantly spaced bunches. In 2011 three filling schemes, which are shown in Figs. 2(a)-2(c), with bunch-to-bunch spacing of 192, 128, and 32 ns were used. The total current was $100 \mathrm{~mA}$ for the schemes with 60 and 240 bunches and $80 \mathrm{~mA}$ in the 40 bunch mode. For a filling scheme with 40 and 60 bunches no phenomena were observed that could be related to EC effects. However, for the filling scheme with 240 bunches ( $32 \mathrm{~ns}$ spacing) a slight vertical emittance growth was observed $\left(\varepsilon_{y} \sim 0.025 \mathrm{~nm}\right)$, which was still acceptable for user operation.

During the startup phase for the 2012 running period, scrubbing runs with 480 equidistant bunches and a total beam current of $100 \mathrm{~mA}$ were done for 4 days. The filling scheme is shown in Fig. 2(d).

During the scrubbing run, phenomena related to EC effects have been observed: horizontal and vertical emittance growth and extra lines in the vertical tune spectra. After the scrubbing run it was possible to operate PETRA III with 240 bunches with a smaller vertical emittance $\left(\varepsilon_{y} \sim 0.01 \mathrm{~nm}\right)$ than in 2011. It was also possible to run with 320 equidistantly spaced bunches and a small vertical emittance growth. But for filling schemes with 480 bunches [Fig. 2(d)] and 960 bunches ( $8 \mathrm{~ns}$ bunch spacing, design parameter), a significant emittance growth was observed, which excludes these filling schemes for user runs.

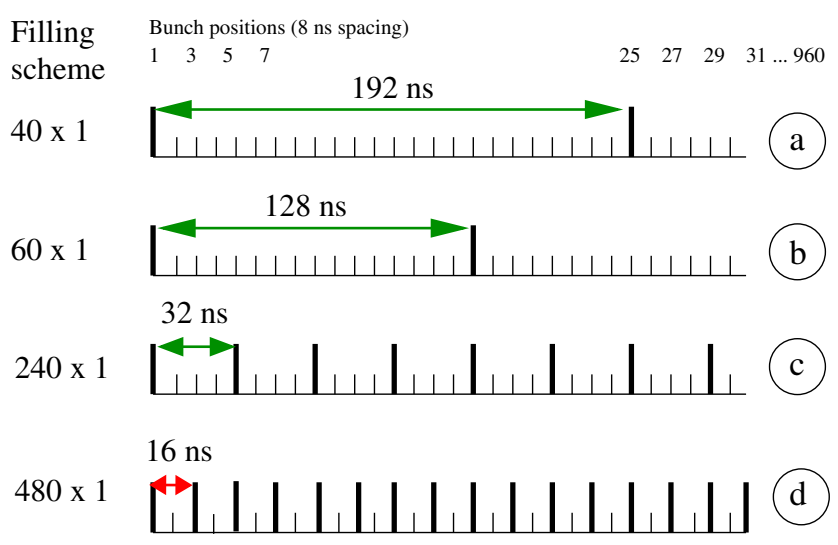

FIG. 2. Bunch filling schemes of PETRA III for the user runs in 2011 (a)-(c) and for the scrubbing run in March 2012 (d). 


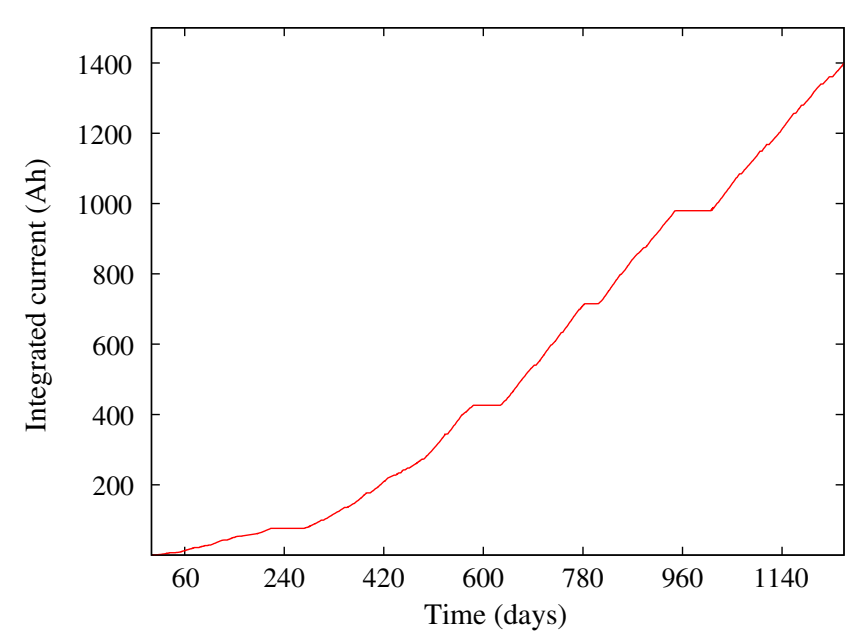

FIG. 3. Integrated beam current of PETRA III. The time scale starts at May 1, 2009, and ends at October 22, 2012.

The integrated beam current of PETRA III is shown in Fig. 3 from May 1, 2009, to October 22, 2012. The first user runs with 240 bunches started in August 2010 with a special scheme with 60 trains with 4 bunches [22]. The integrated beam current was $230 \mathrm{Ah}$. At the beginning of the user runs in February 2011 an integrated beam current of 430 Ah was reached and the filling schemes of Figs. 2(a)-2(c) were used, including the scheme with equidistantly spaced bunches. Aside from the scrubbing runs, in 2012 a conditioning effect based on the integrated beam current was observed.

To have a simple estimate of the number of electrons hitting the accelerator wall during the described operation periods, we first calculate the number of emitted photons per length and per positron in the bunch in the dipole magnet with bending radius $\rho(\sim 192 \mathrm{~m})$ :

$$
\frac{d N_{\gamma}}{d s}=\frac{5}{2 \sqrt{3}} \alpha \frac{E}{m_{0} c^{2}} \frac{1}{\rho}=0.645 \mathrm{~m}^{-1},
$$

where $\alpha(\sim 1 / 137)$ is the fine structure constant and $E$ is the energy of the beam. The electron dose due to photoelectrons and secondary electrons on the surface can be estimated by using the simulation code ECLOUD [17]. Assuming a photoelectron yield $(Y)$ of 0.1 and taking into account a SEY of only 1.5 , one obtains a photoelectron dose of $7.6 \times 10^{-2} \mathrm{C} / \mathrm{mm}^{2}$ in the central region of chamber using the filling scheme $c$ of Fig. 2 and also considering that the integrated current was 9.6 Ah for the scrubbing runs (4 days at $100 \mathrm{~mA}$ ). In the simulations most of the photoelectrons were emitted from the region where the primary synchrotron radiation hits the wall, but a fraction (30\%) was emitted from other parts of the chamber due to the reflectivity of the $\mathrm{Al}$ alloy surface (see also the results for $\mathrm{Cu}$ in Refs. [9,23]). For a SEY of 1.8 one obtains a photoelectron dose of $0.19 \mathrm{C} / \mathrm{mm}^{2}$ in the central region of the chamber. These simple calculations suggest that $0.1-0.2 \mathrm{C} / \mathrm{mm}^{2}$ is a reasonable dose range expected to hit the accelerator walls during operation and scrubbing runs. This defines the necessity of experimentally measuring the SEY of the Petra III sample not only on the asreceived surface but also after an electron dose comparable to the one here estimated. The goal is then to cross-check experimentally if the Al SEY could decrease to values as low as $1.8-1.5$ validating the input parameters used in ECLOUD simulations.

\section{EXPERIMENTAL}

XPS and SEY measurements were performed at the Material Science INFN-LNF laboratory in Frascati (Rome, Italy) under ultrahigh vacuum (UHV) conditions (unbaked system, base pressure $2 \times 10^{-9} \mathrm{mbar}$ ). The UHV system includes a $\mu$-metal chamber, with residual magnetic field at the sample position lower than $5 \mathrm{mG}$, dedicated to SEY measurements and XPS analysis connected to a second chamber used for in situ sample preparation.

The samples used in this work were cut out of a prototype chamber of Petra III used for assembly tests. The prototype chamber, made of the AlMgSi0,5 (6060) alloy, had the same material properties and got exactly the same surface processing [sodium hydroxide and alkaline agent (Grato 90) cleaning procedures, each step followed by high pressure water rinsing] as the chambers that were installed in the accelerator.

The $\operatorname{SEY}(\delta)$ is defined as the number of secondary electrons emitted per incident electron, i.e., the ratio between the number of electrons leaving the surface and the number of incident electrons per unit area. In practice the currents measured experimentally are the primary current hitting the sample $I_{P}$ and the total sample current $I_{T}=$ $I_{P}-I_{S}$, where $I_{S}$ is the secondary electron current leaving the sample, so that $\delta=1-I_{T} / I_{P}$. The maximum value of the SEY is indicated by $\delta_{\max }$ and this parameter is used for the simulations of the EC buildup in the accelerators. For the SEY measurements the electron beam was set to be smaller than $0.25 \mathrm{~mm}^{2}$ in a transverse cross-sectional area and stable in current for energies between 10 and $500 \mathrm{eV}$, as confirmed by a line profile and by stability tests done by using a homemade $1 \mathrm{~mm}$ slot Faraday cup. To measure low-energy impinging primary electrons, a negative bias voltage $(\sim 75 \mathrm{~V})$ was applied to the sample. Such bias allowed us to work also at very low primary energy (close to $0 \mathrm{eV}$ ) while keeping the gun in a region where it was stable and well focused. The $I_{p}$ current was measured by means of a Faraday cup. During SEY acquisition, the gun current was set to low values (a few tens $\mathrm{nA}$ ) not to perturb the surface with the electron beam; we calculated that the maximal electron dose delivered during the most accurate $\delta$ measurements was of the order of $3 \times 10^{-5} \mathrm{C} / \mathrm{mm}^{2}$. The electron dose was determined from the relation $D=$ $Q / A=\left(I_{P} \times T\right) / A$, where $Q$ is the total charge incident on the sample surface, $I_{P}$ is the impinging beam current (generally a few $\mu \mathrm{A}$ ), and $T$ is the irradiation time. The area $A$ was determined by assuming that the electron beam 
spot on the sample is circular. All electron irradiation and SEY measurements were performed at room temperature and at normal incidence. During scrubbing, the beam current was typically a few $\mu \mathrm{A}$ and the electron beam hit the sample in a fixed area of $\sim 1 \mathrm{~mm}^{2}$. In the course of this study, we found the SEY measurements for a given sample condition to fluctuate by $5 \%$ at most.

XPS spectra were obtained by exciting the sample with nonmonochromatic $\operatorname{MgK} \alpha$ photons $(h \nu=1253.6 \mathrm{eV})$ and detecting the photoemitted electrons by means of an Omicron EA125 hemispherical electron analyzer. Photoelectrons were collected in normal emission geometry. The binding energies (BE) are referred to the Fermi level measured on the sample. The area viewed by the electron analyzer $\left(\sim 1.5 \mathrm{~mm}^{2}\right)$ was slightly larger than that of the region effectively modified by the electron beam $\left(\sim 1 \mathrm{~mm}^{2}\right)$. This implied that the collected photoelectrons were not entirely coming from the irradiated area, but for a minimal fraction originated from the surrounding region. Because of that, the effect of the electron irradiation can result slightly underestimated by the XPS spectra.

\section{RESULTS AND DISCUSSION}

\section{A. SEY of the as-received and electron-scrubbed sample}

Figure 4 shows the XPS spectra and the SEY curves recorded in different conditions on the $\mathrm{Al}$ alloy sample cut from the Petra III prototype chamber. The curve (a) in the top panel shows the survey XPS spectrum acquired on the as-received sample, i.e., just after the insertion into the analysis chamber. The most intense peaks are the $\mathrm{O} 1 \mathrm{~s}$ and $\mathrm{C} 1 \mathrm{~s}$ core levels at 532 and $286 \mathrm{eV}$, respectively, due to surface adsorbates related to ambient air, like water, hydrocarbons, and carbonaceous oxides. Such contaminants cover the sample surface and dump the intensity of the $\mathrm{Al} 2 \mathrm{p}$ and Al2s peaks, which are slightly visible at BEs of 75 and $120 \mathrm{eV}$, typical of oxidized aluminum [24]. A negligible contamination due to $\mathrm{Ca}$ atoms is also observed at about $350 \mathrm{eV}$.

The SEY curve measured on an as-received sample and shown in the bottom panel of Fig. 4 (black line) exhibits a $\delta_{\max }$ value of 2.7. This high value can be attributed to the surface contaminating compounds that are known to increase the secondary electron yield $[15,25,26]$.

In order to simulate the electron conditioning in the accelerator environment, the sample was irradiated for increasing time by an electron beam at kinetic energy of $500 \mathrm{eV}$. Subsequent irradiation cycles progressively reduced the SEY down to a final $\delta_{\max }$ value of 1.8 (red curve in Fig. 4) obtained for a cumulative electron dose of $1.2 \times 10^{-1} \mathrm{C} / \mathrm{mm}^{2}$. In principle the electron scrubbing acts as an electron stimulated desorption process, which modifies the chemistry of the topmost layers and partially removes the surface contaminants [27]. This action usually reflects in a sizeable decrease of the whole SEY curve, and
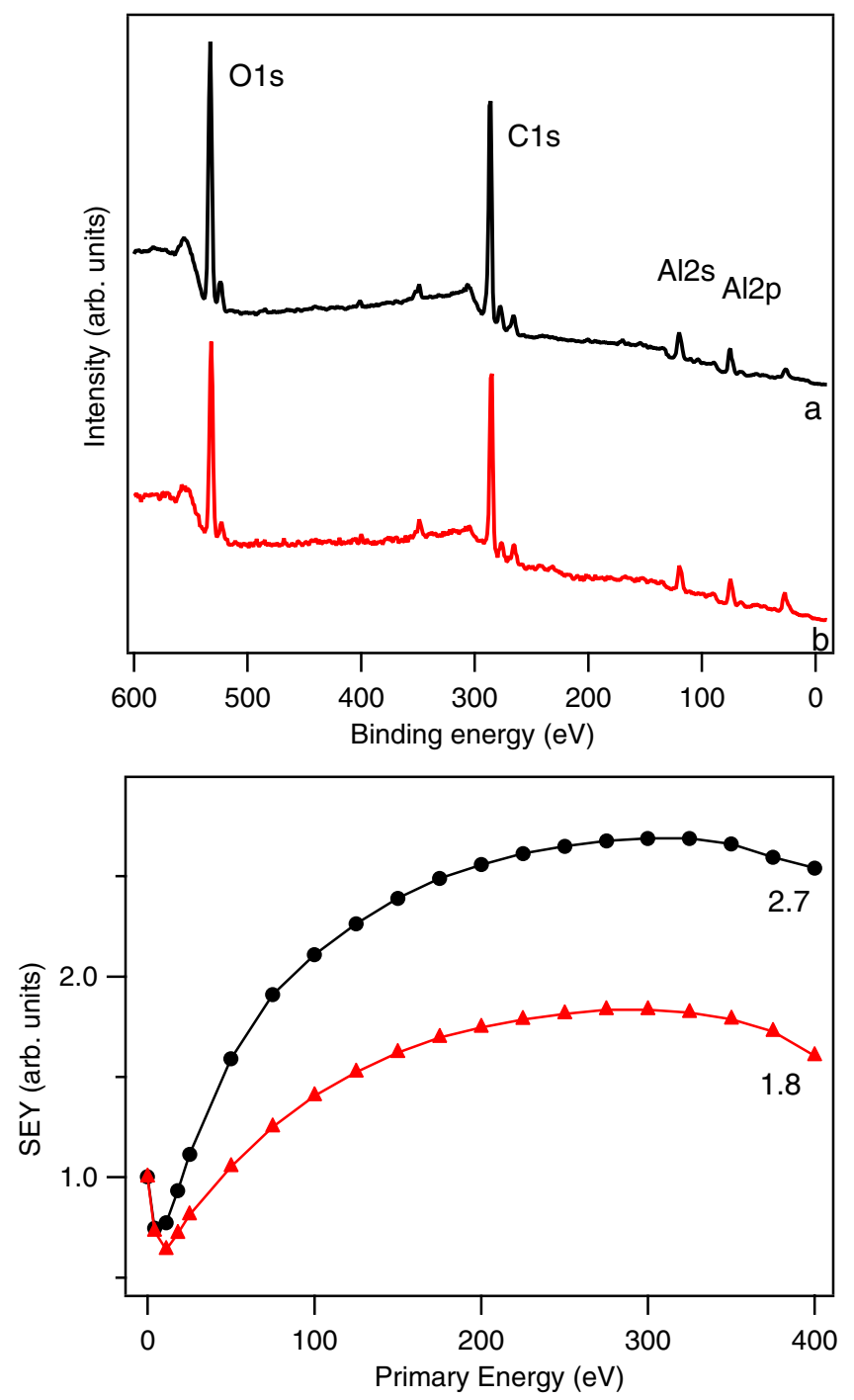

FIG. 4. Top: XPS survey spectra; bottom: SEY curves vs electron beam energy measured on the PETRA III Al sample. (a), (black) as-received and (b), (red) after $e^{-}$beam irradiation, $500 \mathrm{eV}, 1.2 \times 10^{-1} \mathrm{C} / \mathrm{mm}^{2}$. The numbers in the bottom panel report the $\delta_{\max }$ value of each curve.

indeed several technical surfaces in their "fully scrubbed" state meet the requirement compatible with machine operation [28]. In this case, however, the irradiation time (electron dose) needed to reduce the SEY to acceptable values for PETRA III normal operation is significantly longer than any reasonable value, and this renders such EC mitigation scheme of limited efficiency. We show here that this is due to the fact that the chemical state of the surface is not affected significantly by the prolonged electron irradiation. In fact, the XPS spectrum measured on the scrubbed sample shown by the curve (b) in Fig. 4 indicates that the composition of the surface layer has not been noticeably modified. The only change evident with respect to the as-received spectrum consists in the decrease of both $\mathrm{O} 1 \mathrm{~s}$ and $\mathrm{C} 1 \mathrm{~s}$ intensities with a preferential desorption of 
oxygen with respect to carbon; indeed the $\mathrm{O} 1 \mathrm{~s}$ and $\mathrm{C} 1 \mathrm{~s}$ spectral intensities measured on the scrubbed surface were $22 \%$ and $14 \%$ lower, respectively, than those of the asreceived surface. The $\delta_{\max }$ value of 1.8 measured on the scrubbed surface is consistent with those reported for experiments performed by electron bombarding similar $\mathrm{Al}$ alloy samples $[14,15]$. In contrast the $\delta_{\max }$ value measured on the sputter cleaned $\mathrm{Al}$ alloy 6061 surface is around 1 [6].

The decrease of $C$ and $O$ intensities suggests that contaminating adsorbates are partially desorbed by the electron beam and the variation of $\delta_{\max }$ from 2.7 to 1.8 indicates that these species gave a high contribution to the SEY. What is left after the scrubbing is basically the same $\mathrm{Al}$ oxidized surface with a lower amount of O-carrying contaminants on top. The fact that the line shape of the Al core levels does not change appreciably after scrubbing could indicate that most of the desorbed species were not directly bonded to $\mathrm{Al}$, and that the $\mathrm{Al}$ oxide layer is not decomposed by the electron beam. However, it is also possible that residual gas adsorbates $\left(\mathrm{H}_{2} \mathrm{O}, \mathrm{CO}, \mathrm{CO}_{2}\right)$ dissociated by the electrons replace the desorbed $\mathrm{O}$ (and $\mathrm{C}$ ) atoms, so that on the average we do not see any substantial change. The balance between the electron beam-induced desorption and recontamination, which determines the ultimate $\delta_{\max }$ measured after scrubbing, depends on the value and composition of the residual gas pressure in the UHV chamber where the electron conditioning is performed. The vacuum environment where the experiment was performed, that is an unbaked system with a background pressure of $2 \times 10^{-9}$ mbar dominated by the $\mathrm{H}_{2} \mathrm{O}$ partial pressure $\left[p_{\mathrm{H}_{2} \mathrm{O}}(m=18)=5 \times 10^{-10}\right.$ mbar] [29] (see Supplemental Information [30]) is representative for the PETRA III vessel, which was only prebaked prior to installation and whose typical pressure with beam running is about $3 \times 10^{-9} \mathrm{mbar}$. The role of the background pressure and residual gas composition in the evolution of the SEY of the $\mathrm{Al}$ alloy sample with electron scrubbing becomes clear when comparing the results reported above with those obtained by electron scrubbing a similar PETRA III sample in a second cryo-pumped, baked UHV chamber with a base pressure of $1 \times 10^{-10}$ mbar dominated by the mass peak due to hydrogen $\left[p_{\mathrm{H}_{2} \mathrm{O}}(m=18)=2 \times 10^{-11}\right.$ mbar] [29] (see Supplemental Information [30]). In this case an equivalent dose of $1.2 \times 10^{-1} \mathrm{C} / \mathrm{mm}^{2}$ at $500 \mathrm{eV}$ reduced $\delta_{\max }$ to values as low as 1.5 [5]. We want to stress that the main and this second UHV systems were almost-twin, having similar shape and volume, and were equipped with identical $e$-guns, vacuum gauges, and Faraday cups. It can be reasonably excluded that the different $\delta_{\max }$ values measured in the two UHV systems could be due to different geometry or focusing conditions. As it will be better shown below, this proves the dramatic reactivity of the Al surface to adventitious adsorbates that might result even enhanced under electron beam irradiation.

For other technical surfaces as TiN and $\mathrm{Cu}[6-8,12,13]$ it has been observed that electron conditioning reduces the high $\delta_{\max }$ values measured on the as-received samples to values of the order of 1.3 or even lower, and this behavior has been related to the desorption of contaminants as well as to the formation of a graphitic film upon the interaction of the electron beam with $\mathrm{C}$ containing surface adsorbates. For the PETRA III Al alloy sample, however, in spite of the large quantity of $\mathrm{C}$ at the surface, a similar growth of a graphitic layer has not been observed. The $\mathrm{C} 1 \mathrm{~s}$ peak measured on the scrubbed surface, besides the limited intensity decrease mentioned above, maintains the pristine broad profile centered at $285.6 \mathrm{eV}$, indicating the presence of a disordered assembly of $\mathrm{C}$ atoms involved in C-C, C-H, and $\mathrm{C}-\mathrm{O}$ bonds. The stability of the $\mathrm{C} 1 \mathrm{~s}$ line shape excludes a reorganization of the $\mathrm{C}$ atoms into a $s p^{2}$ graphitic network, that would contribute at BEs more than $1 \mathrm{eV}$ lower [31], and thus determine a shift of the measured $\mathrm{C} 1 \mathrm{~s}$ spectrum.

\section{B. Effect of the surface oxidation on SEY}

In order to evaluate the effect of the adsorbate contamination on the SEY properties we cleaned the Al alloy surface by $\mathrm{Ar}^{+}$sputtering. In the XPS spectrum measured on the ion bombarded sample, which is shown by the curve (a) in Fig. 5, the O1s peak has halved its intensity, the $\mathrm{C}$ contamination is almost eliminated, and the intensity of the $\mathrm{A} 12 \mathrm{~s}$ and Al2p core levels appears strongly enhanced. The presence of negligible extra peaks due to nitrogen $(397 \mathrm{eV})$ and tantalum $(240 \mathrm{eV}$ and $238 \mathrm{eV})$ can be attributed to native metal contamination and to the sputtering of the sample holder, respectively. The SEY measured on the $\mathrm{Ar}^{+}$ sputtered $\mathrm{Al}$ surface (green curve in Fig. 5) is strongly reduced showing a $\delta_{\max }$ value of 1.3 , which approaches the value of the clean metal alloy [6].

The high resolution Al2p spectrum measured on the $\mathrm{Ar}^{+}$ sputtered surface is shown at the bottom of Fig. 6. Because of the low percentage of the other alloy components ( $\mathrm{Si}$ and $\mathrm{Mg} \leq 0.6 \mathrm{wt} \% ; \mathrm{Fe} \leq 0.3 \mathrm{wt} \% ; \mathrm{Zn}, \mathrm{Cu}, \mathrm{Mn}, \mathrm{Ti} \leq$ $0.15 \mathrm{wt} \%$ ) the spectrum was decomposed by considering $\mathrm{Al}-\mathrm{Al}$ bonds and $\mathrm{Al}$ atoms coordinated with chemisorbed oxygen or organized in oxide phases [24,32-34]. The spectra were best fitted with Voigt functions by using four doublet components, the first at $\mathrm{BE}\left(2 p_{3 / 2}\right)$ of $72.5 \mathrm{eV}(\mathrm{C} 1)$ due to metallic $\mathrm{Al}$ and the others at $73.4 \mathrm{eV}$ (C2), $73.9 \mathrm{eV}(\mathrm{C} 3)$, and $75.1 \mathrm{eV}(\mathrm{C} 4)$. In agreement with the literature $\mathrm{C} 2$, which has a chemical shift of $0.9 \mathrm{eV}$ with respect to $\mathrm{C} 1$, is attributed to $\mathrm{Al}$ atoms bonded to chemisorbed $\mathrm{O}$ atoms [24] and $\mathrm{C} 3$ and $\mathrm{C} 4$ shifted by 1.4 and $2.6 \mathrm{eV}$ to tetrahedral and octahedral $\mathrm{Al}_{2} \mathrm{O}_{3}$ phases, respectively $[32,33]$.

The Al2p core level analysis shows that after prolonged ion bombardment there are still $\mathrm{Al}$ atoms bonded to oxygen even in a $\mathrm{Al}_{2} \mathrm{O}_{3}$ environment, indicating the difficulty to remove completely the $\mathrm{Al}$ oxides in the $2 \times 10^{-9} \mathrm{mbar}$ background pressure of our system. This is due to the high propensity of $\mathrm{Al}$ to oxidize even at room temperature: the minimal partial pressure of water contained in the residual 

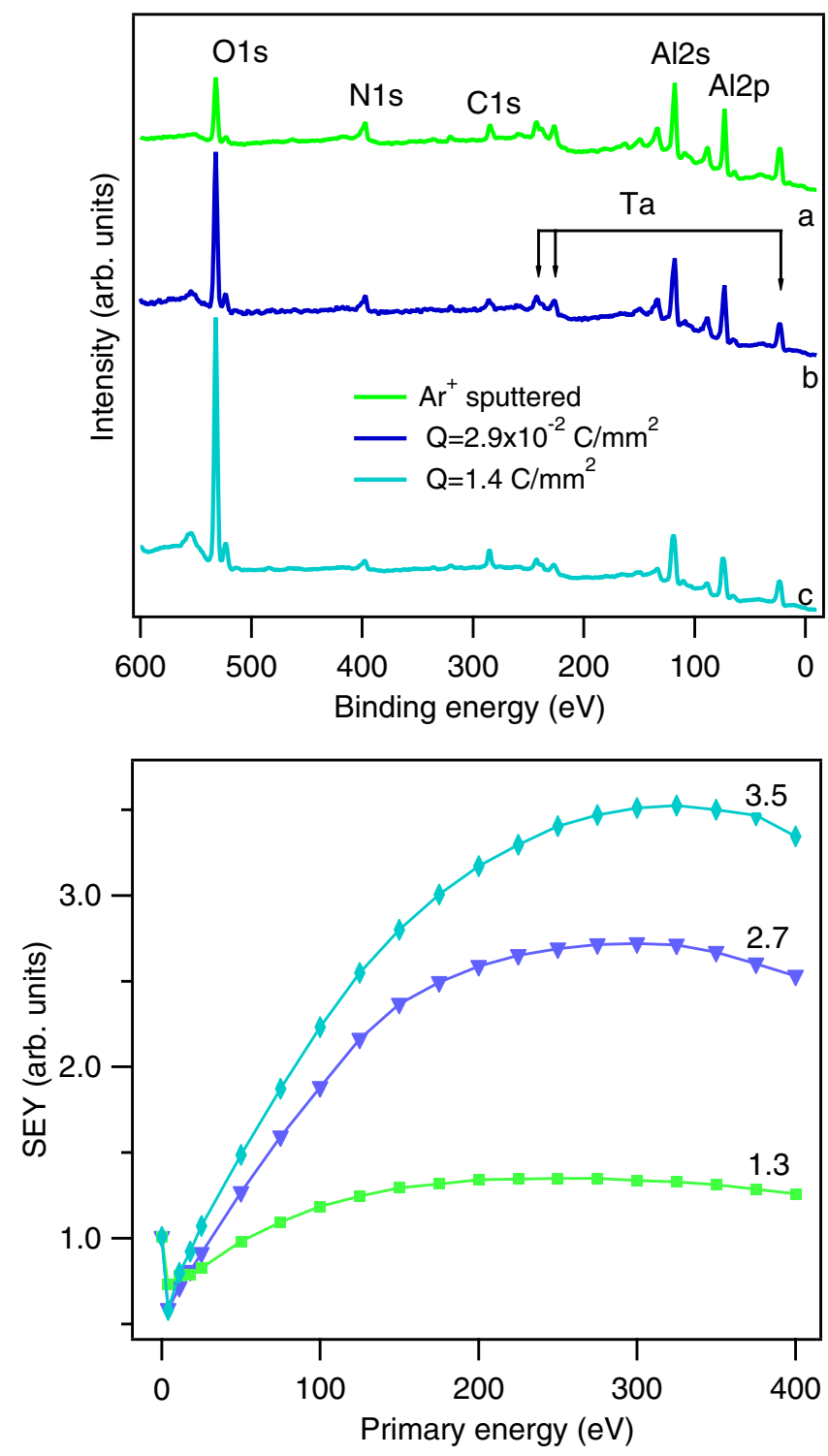

FIG. 5. Top: XPS survey spectra; bottom: SEY curves vs electron beam energy measured on the PETRA III Al sample. (a), (green) after $\mathrm{Ar}^{+}$bombardment at $2 \mathrm{KeV}$; (b), (blue) after $e^{-}$ beam irradiation, $500 \mathrm{eV}, 2.9 \times 10^{-2} \mathrm{C} / \mathrm{mm}^{2}$; (c), (cyan) after $e^{-}$beam irradiation, $500 \mathrm{eV}, 1.4 \mathrm{C} / \mathrm{mm}^{2}$. The numbers in the bottom panel report the $\delta_{\max }$ value of each curve.

gas was sufficient to hinder the achievement of a stable, clean Al alloy surface. Such surface oxide phases are responsible for the difference between the measured $\delta_{\max }$ value of 1.3 and the value of 1 expected for the clean surface [6].

We proved the strong role of the surface oxygen in increasing the SEY by oxidizing on purpose the $\mathrm{Al}$ alloy surface by electron beam irradiation. In fact, due to the extremely high affinity of $\mathrm{Al}$ towards $\mathrm{O}$ radicals, the dissociation of a residual gas molecule as $\mathrm{H}_{2} \mathrm{O}$ and $\mathrm{CO}$ induced at the metal surface by the electron beam determines a rapid oxidation of the spot area hit by the beam, as

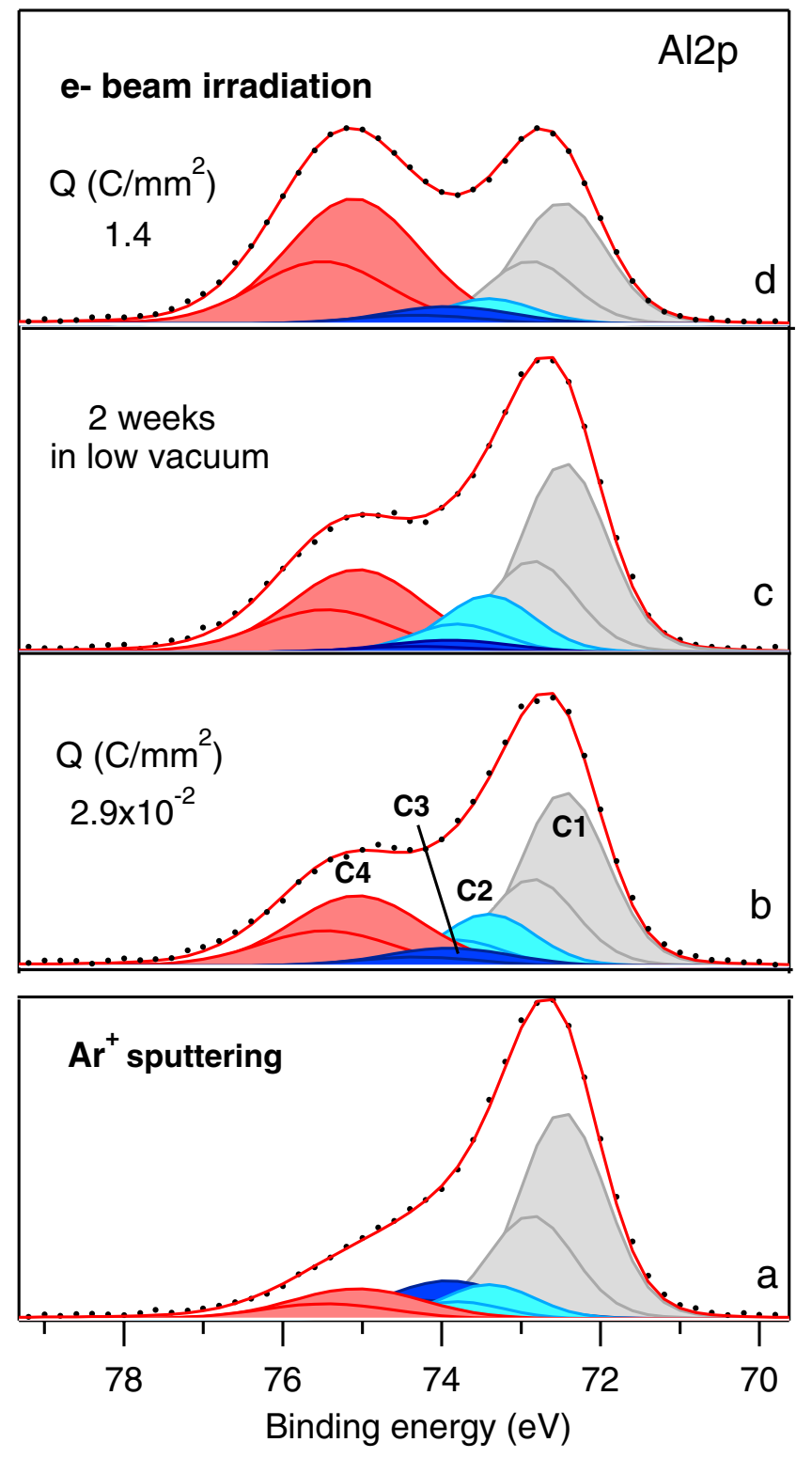

FIG. 6. Al2p spectra measured on the Al PETRA III sample (a) after ion sputtering at $2 \mathrm{KeV}$ and after electron beam irradiation at $500 \mathrm{eV}$ up to a dose of (b) $2.9 \times 10^{-2} \mathrm{C} / \mathrm{mm}^{2}$ and (d) $1.4 \mathrm{C} / \mathrm{mm}^{2}$. (c) Spectrum taken on the dosed sample (b) after two weeks of permanence in low vacuum $\left(1 \times 10^{-4} \mathrm{mbar}\right)$. The experimental spectra (black circles) are shown together with the best fit curves (red lines) and the $\mathrm{C} 1-\mathrm{C} 4$ doublet components (see text). Spectral decomposition was obtained by considering a spin orbit splitting of 0.4 and a branching ratio of 2 between the $3 / 2$ and $1 / 2$ doublet components.

well as, although to a lesser extent, of the surrounding region.

The XPS spectra measured on the surface exposed to electron doses of $2.9 \times 10^{-2}$ and $1.4 \mathrm{C} / \mathrm{mm}^{2}$ at $500 \mathrm{eV}$ and shown by the curves (b) and (c) in Fig. 5, respectively, indicate a progressive increase of the $\mathrm{O} 1 \mathrm{~s}$ peak in contrast to the constant intensity of the $\mathrm{C} 1 \mathrm{~s}$ peak, that keeps the 
value measured after ion bombardment. In agreement with the O1s increase, the Al2p spectra in Fig. 6 show, after the first irradiation [Fig. 6(b)], a weak increase of the $\mathrm{C} 2$ as well as of the C4 component, and later [Fig. 6(d)] a dramatic enhancement exclusively of the most oxidized $\mathrm{Al}_{2} \mathrm{O}_{3}$ phase. Accordingly $\delta_{\max }$ rises to 2.7 after the first and to 3.5 after the second electron irradiation, the increase being due to the growth of $\mathrm{Al}_{2} \mathrm{O}_{3}$ [35]. We want to point out that between the first and the second irradiation the sample was kept for two weeks in low vacuum $\left(1 \times 10^{-4}\right.$ mbar $)$ to probe the rate of $\mathrm{Al}$ oxidation without the action of the electron beam. We found that the permanence of the sample in low vacuum was ineffective to more heavily oxidize the surface. Indeed, the survey XPS spectrum (not shown) as well as the high resolution A12p spectrum [see Fig. 6(c)] were very similar to those measured two weeks before [Fig. 6(b)]. This proves the strong effect that electron irradiation has on the oxidation rate and therefore on the $\delta$ increase of the Al surface.

The relation between the SEY and the oxidation state of the surface is summarized in Fig. 7, where the intensity of the O1s peak, as well as of the $\mathrm{C} 4$ component of the Al2p core level, are compared with the $\delta_{\max }$ values after each of the treatments described above. The comparison clearly shows that the SEY variation follows the oxygen content of the $\mathrm{Al}$ alloy surface. These results disclose an unfavorable scenario for the possibility to achieve electron beam conditioning of the inner wall of PETRA III, as well as of other accelerator chambers made of $\mathrm{Al}$ alloys.

Because of the high chemical propensity of $\mathrm{Al}$ towards oxygen and to the high SEY of the $\mathrm{Al}_{2} \mathrm{O}_{3}$ phases, each chemical reaction producing oxidizing radicals in the environment surrounding the $\mathrm{Al}$ surface unavoidably binds $\mathrm{O}$ atoms to the metal surface and increases the $\delta_{\max }$ value.

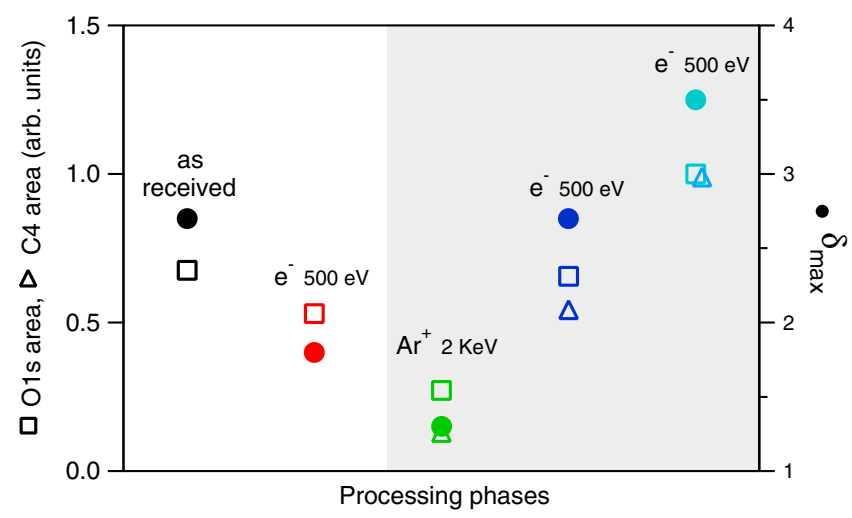

FIG. 7. O1s intensity (squares), $\mathrm{Al}_{2} \mathrm{O}_{3}$ (C4) intensity (triangle), and $\delta_{\max }$ (circles) measured on the Petra III Al sample after different surface treatments comprised in two experiments that are the irradiation of the as-received $\mathrm{Al}$ alloy (left part) and the irradiation of the sample cleaned by $\mathrm{Ar}^{+}$sputtering (right part); (black) as-received; (red) $e^{-}$beam, $500 \mathrm{eV}, 1.2 \times$ $10^{-1} \mathrm{C} / \mathrm{mm}^{2}$; (green) $\mathrm{Ar}^{+}$bombardment, $2 \mathrm{KeV}$; (blue) $e^{-}$ beam, $500 \mathrm{eV}, 2.9 \times 10^{-2} \mathrm{C} / \mathrm{mm}^{2}$; (cyan) $e^{-}$beam, $500 \mathrm{eV}$, $1.4 \mathrm{C} / \mathrm{mm}^{2}$.
The evidence we obtained that $\mathrm{C}$ does not accumulate on the surface under electron beam irradiation at $500 \mathrm{eV}$ tends to discard the possibility that electron scrubbing could generate a thin $\mathrm{C}$-based passivating layer on $\mathrm{Al}$ alloys, as happens in the case of other metals [7,36]. The conclusion of this study is that radiation conditioning inside Petra III might not be successful in decreasing the $\delta_{\max }$ to values lower than 1.5. Where lower SEY are desired it seems that the only possibility to use $\mathrm{Al}$ vacuum chambers for accelerator rings is to coat the inner walls with a stable thin film of a low $\delta_{\max }$ material, suitable to hinder the interaction of the Al surface with the oxidizing species that inevitably develop during machine running. A similar strategy was also suggested by preliminary studies performed in the Positron low-energy ring of the PEP-II accelerator [8].

\section{CONCLUSIONS}

We measured the SEY curves and studied the effects of electron conditioning for $\mathrm{Al}$ alloy samples cut from the dipole chamber of PETRA III at DESY. X-ray photoelectron spectroscopy gives us a way to understand the chemical origin of the observed $\delta_{\max }$ variations. Our measurements show that the SEY decrease upon electron scrubbing at $500 \mathrm{eV}$ is limited, and the final $\delta_{\max }$ value ranging between 1.8 and 1.5 might not be sufficiently low to prevent EC effects. This is consistent with simulations done for different filling patterns indicating that the SEY is below 2.0 in PETRA III after conditioning and scrubbing in 2012.

Our experimental study shows that such value is indeed possible to be obtained after scrubbing. Also the dose necessary to reach such SEY values is consistent to the one received by the accelerator walls during last year operation, as estimated by simple calculations. We than confirm that the minimum SEY value of Al alloy of Petra III can indeed be assumed to range between 1.5 and 1.8 after scrubbing, and that the calculation used to demonstrate the importance of EC phenomena for the emittance growth of Petra III are based on reasonable parameters.

The analysis of the chemical mechanism governing the scrubbing for such Al alloys shows that the SEY of the samples is severely affected by the presence of surface contaminating species. Indeed we find that the $\mathrm{Al}$ alloy surface is very sensitive to experimental conditions (like residual pressure and its composition, gun degassing, etc.), and this could explain the spread in the $\delta_{\max }$ measured from different samples and in different UHV environments. We demonstrate the predominant role of the $\mathrm{Al}_{2} \mathrm{O}_{3}$ phase in rising the SEY. Moreover we show that the $\mathrm{Al}_{2} \mathrm{O}_{3}$ growth is promoted by the residual gas cracking induced by electron irradiation at $500 \mathrm{eV}$. This evidence indicates the limited usefulness of electron scrubbing for achieving inner wall conditioning of $\mathrm{Al}$ alloys. The use of coated or otherwise treated $\mathrm{Al}$ walls seems to be the most promising possibility to face the EC problem. 


\section{ACKNOWLEDGMENTS}

This work was partially supported by INFN-NTA funding agency, within the IMCA project. The assistance of the technical team of DAFNE-L is deeply acknowledged. We thank the group MVS at DESY for providing the $\mathrm{Al}$ samples and the photograph of the dipole vacuum chamber. R. W. would like to thank K. Balewski, A. Kling, and G. K. Sahoo for valuable discussions.

[1] R. Cimino, I. Collins, M. Furman, M. Pivi, F. Ruggiero, G. Rumolo, and F. Zimmermann, Phys. Rev. Lett. 93, 014801 (2004).

[2] J. Wang, Z. Guo, Y. Liu, Q. Qin, J. Xing, and Z. Zhao, Phys. Rev. ST Accel. Beams 7, 094401 (2004).

[3] K. Harkay and R. Rosenberg, Phys. Rev. ST Accel. Beams 6, 034402 (2003).

[4] M. Izawa, Y. Sato, and T. Toyomasu, Phys. Rev. Lett. 74, 5044 (1995).

[5] D. R. Grosso, M. Commisso, R. Cimino, R. Flammini, R. Larciprete, and R. Wanzenberg, Proceedings of IPAC2011, San Sebastián, Spain (EPS-AG, Spain, 2011), p. 1533, TUPS009.

[6] V. Baglin, J. Bojko, O. Gröbner, B. Henrist, N. Hilleret, C. Scheuerlein, and M. Taborelli, Proceedings of the 7th European Particle Accelerator Conference, Vienna, Austria CERN-LHC-Project-Report No. 433, pp. 217-221.

[7] M. Nishiwaki and S.Kato, Vacuum 84, 743 (2009).

[8] M. T.F. Pivi, G. Collet, F. King, R.E. Kirby, T. Markiewicz, T. O. Raubenheimer, J. Seeman, and F. Le Pimpec, Nucl. Instrum. Methods Phys. Res., Sect. A 621, 47 (2010).

[9] R. Cimino, Nucl. Instrum. Methods Phys. Res., Sect. A 561, 272 (2006).

[10] R. Cimino, I. R. Collins, and V. Baglin, Phys. Rev. ST Accel. Beams 2, 063201 (1999).

[11] R. Cimino, M. Commisso, T. Demma, D. R. Grosso, N. Mahne, and A. Giglia, Proceedings of ECLOUD10, Cornell University, Ithaca, New York, 2010, p. 46, MIT03.

[12] R. Cimino, M. Commisso, D. R. Grosso, T. Demma, V. Baglin, R. Flammini, and R. Larciprete, Phys. Rev. Lett. 109, 064801 (2012).

[13] R. Larciprete, D. R. Grosso, M. Commisso, R. Flammini, and R. Cimino, Phys. Rev. ST Accel. Beams 16, 011002 (2013).

[14] R. Rosenberg, M. W. McDowell, Q. Ma, and K. Harkay, J. Vac. Sci. Technol. A 21, 1625 (2003).

[15] F. Le Pimpec, R. E. Kirby, F. King, and M. Pivi, J. Vac. Sci. Technol. A 23, 1610 (2005).

[16] Y. Lin and D. C. Joy, Surf. Interface Anal. 37, 895 (2005).
[17] G. Rumolo and F. Zimmerman, CERN Report No. CERNSL-Note-2002-016 (AP).

[18] DESY Report ISSN 0418-9833, DESY 2004-035, and http://petra3-project.desy.de/general/tdr/index_eng.html.

[19] M. Seidel et al., Proceedings of the 21st Particle Accelerator Conference, Knoxville, 2005 (IEEE, Piscataway, NJ, 2005), p. 2473.

[20] R. Böspflug et al., J. Phys. Conf. Ser. 100, 092012 (2008).

[21] K. Balewski, Proceedings of the First International Particle Accelerator Conference, Kyoto, Japan, 2010 (IPAC'10 Organizing Committee, Asian Committee for Future Accelerators, Kyoto, 2010).

[22] R. Wanzenberg, DESY Report No. DESY 11-140, 2011.

[23] N. Mahne, V. Baglin, I. R. Collins, A. Giglia, L. Pasquali, M. Pedio, S. Nannarone, and R. Cimino, Appl. Surf. Sci. 235, 221 (2004).

[24] C. F. McConville, D. L. Seymour, D. P. Woodruff, and S. Bao, Surf. Sci. 188, 1 (1987).

[25] B. Henrist, N. Hilleret, C. Scheuerlein, and M. Taborelli, Appl. Surf. Sci. 172, 95 (2001).

[26] N. Hilleret, C. Scheuerlein, and M. Taborelli, Appl. Phys. A 76, 1085 (2003).

[27] M. Ding and E. Williams, Vacuum 39, 463 (1989).

[28] F. Zimmermann, CERN Report No. CERN-SL-2001-003 DI, 2001.

[29] The $\mathrm{H}_{2} \mathrm{O}$ partial spectra were derived by the mass spectra calibrated with the total pressure values as measured by hot filament vacuum gauges. We did not perform any additional calibration to account for the different ionization cross sections of the different molecules in the residual gas. This can generate some error on the absolute values of the $\mathrm{H}_{2} \mathrm{O}$ partial pressures of the two systems, without affecting significantly their ratio as the mass spectra and the total pressures were measured in the two systems with identical devices.

[30] See Supplemental Information at http://link.aps.org/ supplemental/10.1103/PhysRevSTAB.16.051003 for residual gas analysis in the two UHV systems used for the scrubbing experiments of the PETRA III Al alloy samples.

[31] J. Diaz, G. Paolicelli, S. Ferrer, and F. Comin, Phys. Rev. B 54, 8064 (1996).

[32] A. Mulligan, V. Dhanak, and M. Kadodwala, Langmuir 21, 8312 (2005).

[33] A. Bianconi, R. Z. Bachrach, and S. B. M. Hagstrom, Phys. Rev. B 19, 2837 (1979).

[34] C. Berg, S. Raaen, A. Borg, J. N. Andersen, E. Lundgren, and R. Nyholm, Phys. Rev. B 47, 13063 (1993).

[35] M. Belhaj, Th. Tondu, and V. Inguimbert, Appl. Surf. Sci. 257, 4593 (2011).

[36] M. Nishiwaki and S. Kato, J. Vac. Sci. Technol. A 25, 675 (2007). 